\title{
Study the Effect of SRRs on Broadband Microwave Parallel-Coupled Band-Pass Filters
}

\author{
Ahmed Hameed Reja, Syed Naseem Ahmad, and Mushtaq A. Alqaisy
}

\begin{abstract}
In this paper, broadband microwave parallel-coupled band-pass filters for wireless microwave technology are presented. Two designs of filters with center frequency of $10.5 \mathrm{GHz}$ and $9.5 \mathrm{GHz}$ are proposed. A metallic split ring resonator (SRR) as a basic building block for planar filters with dual-band band-pass frequency response, which exhibits simultaneously negative permittivity and permeability as a metamaterial without resorting to additional metallic wires is numerically investigated. The effect of SRRs on these filters to get new two metamaterial dual-band microwave filters is also presented. The dimensions of the second filter are reduced compared to the first filter with better results. Numerical results for all designs are obtained, then the filters are simulated using computer aided design (CAD) in microwave applications (Ansoft HFSS) and implemented on the Roger RT/duroid 6010, dielectric constant $\varepsilon_{r}=10.2$, and a substrate height $t=0.635 \mathrm{~mm}$.
\end{abstract}

Index Terms-Metamaterial, microwave filter, parallel-coupled, split ring resonators.

\section{INTRODUCTION}

The band-pass filter (BPF) has many applications in a microwave system. It serves well in reducing the noise and harmonic content of a system by limiting the band of frequencies seen by the system. Many times, band-pass filters are placed at the front end of a receiver to decrease the out of band noise that can enter. They can be used at the output of a transmitter to reduce or eliminate harmonics and spurious outputs generated within the system [1]. A band-pass filter passes a specific band of frequencies and rejects frequencies below and above that band. The response curve of a band-pass filter is shown in Fig. 1. This response shows an area that is termed the pass-band, which is the area where there is a minimum loss in the filter response [2]. The ratio of the power delivered from a source to a load with and without a two-port network inserted in between is known as the insertion loss of that two-port. The fraction of the input power that is lost due to reflection at its input port is called the return loss. The ratio of the power delivered to a matched load to that supplied to it by a matched source is called the attenuation of that two-port network [3].

Manuscript received July 31, 2013; revised Novemeber 5, 2013.

Ahmed Hameed Reja is with Jamia Millia Islamia, New Delhi, India (e-mail: ahmad8171@yahoo.com).

Syed Naseem Ahmad is with Electronics \& Communication Engineering Department, Jamia Millia Islamia, New Delhi, India (e-mail: snahmad@jmi.ac.in).

Mushtaq A. Alqaisy is with Electronics \& Communication Engineering Department, Universiti Tenaga Nasional, Malaysia (e-mail: mushtaq_alqaisy@yahoo.com).

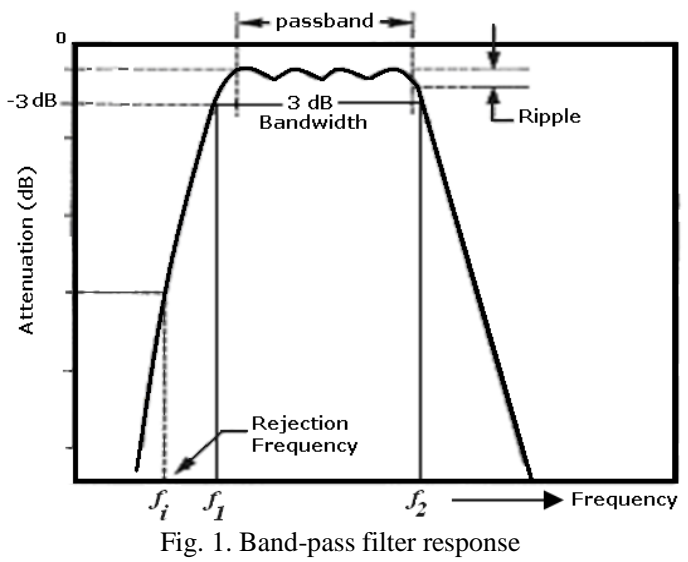

Metamaterials (MTMs) are defined as artificial (fabricated by human), effectively homogeneous ( $p<<\lambda_{g}$ ) and exhibiting highly unusual properties $\left(\varepsilon_{r}, \mu_{r}<0\right)$ not readily available in nature, where $p$ is the average cell size and it is much smaller than the guided wavelength $\left(\lambda_{g}\right)$ [4].

Split-ring resonators (SRR) were one of the first particles proposed for metamaterial construction. Metallic metamaterials comprising double split-ring resonators (SRRs) are the main artificial structures to realize magnetic responses at an electromagnetic spectrum above gigahertz frequencies [5]-[8]. The realization of backward wave propagation using split ring resonator (SRR) and thin wire (TW) and several other electrically small resonators was considered by Pendry et al.[5], [9]. The split ring resonator shown in Fig. 2 is used to obtain a negative value of effective permeability over a desired frequency range.

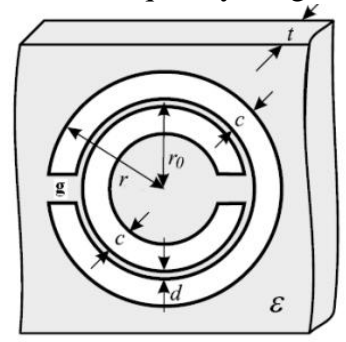

(a) (b)

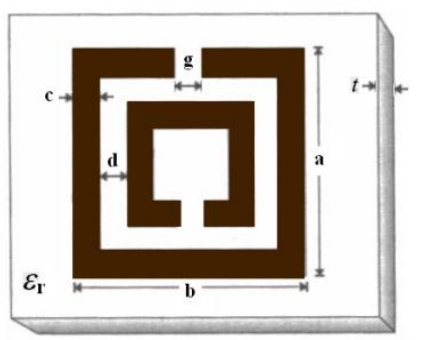

Fig. 2. SRR topology (a) Circle shape, (b) Square shape. The relevant dimensions are indicated.

A kind of LHM, consisting of split ring resonators (SRRs) and continuous wires, whose properties were not determined by the fundamental physical properties of their constituents but the shape and distribution of specific patterns included, was fabricated by Shelby et al. [10] based on Pendry's research. The idea for the implementation of the first MTM TL based on SRRs has been presented by Martin et al. [11]. The SRRs has negative permeability at specific resonance frequency, which can be proved mathematically. This negative permeability can prevent wave propagation at the 
resonant frequency because wave direction is reverse.

In addition to the capability of SRR to generate a negative permeability $\left(\mu_{\text {eff }}<0\right)$, the SRR should be of capability to produce a negative permittivity $\left(\varepsilon_{\text {eff }}<0\right)$ at certain frequencies instead of cut wires. Consequently, a metamaterial composed of SRRs could be an LHM when the negative permeability and permittivity are modulated to a common frequency band. However, this is typically difficult to design [12], [13].

In fact the presence of SRRs in the active region of the filters affects somewhat the electrical characteristics of the structures. It has been found that the presence of SRRs in the parallel coupled-line filters modifies the characteristics of the coupled-line sections as compared to the structures without SRRs. Therefore, it is necessary to recalculate dimensions to preserve the central filter frequency and bandwidth [14].

Parallel-coupled microstrip filters have been widely used for decades in the RF front end of wireless communication systems. The design method for parallel-coupled microstrip filters is presented in [15], [16]. There are many published papers on parallel-coupled band-pass filters using different techniques and different frequencies [17]-[20].

In this paper, four broadband parallel-coupled microwave filters are proposed. The first design operates at $10.5 \mathrm{GHz}$ center frequency and has pass-band of (8-13) $\mathrm{GHz}$ with minimum attenuation of $-37.5 \mathrm{~dB}$ at $1.4 \mathrm{GHz}$. The specifications of Broadband filter are given in the Table I.

The designed filter is used in microwave wireless applications such as satellite television receivers. The design of filters using Ansoft HFSS software design and implemented on Roger RT/duroid, PTFE substrate with dielectric constant $\varepsilon_{r}=10.2$, loss tangent $\tan \alpha=0.0023$ and substrate height $t=0.635 \mathrm{~mm}$.

TABLE I: BROADBAND PASS FILTER DESIGN SPECIFICATION

\begin{tabular}{|c|c|}
\hline Filter specifications & Values \\
\hline Center frequency, $f_{o}$ & $10.5 \mathrm{GHz}$ \\
\hline Upper cut-off frequency, $f_{2}$ & $13 \mathrm{GHz}$ \\
\hline Lower cut-off frequency, $f_{1}$ & $8 \mathrm{GHz}$ \\
\hline Bandwidth & $5 \mathrm{GHz}$ \\
\hline Minimum attenuation & $-37.5 \mathrm{~dB}$ at $1.4 \mathrm{GHz}$ \\
\hline
\end{tabular}

To obtain a new band-pass filter, the above designed filter is modified using $\mathrm{U}$-shape resonator which miniaturizes the dimensions and gives better results at $9.5 \mathrm{GHz}$ center frequency. The effects of SRRs on filters to get dual-band filter around center frequency are also presented. In addition, a left-handed response with simultaneously negative permittivity $\left(\varepsilon_{e f f}\right)$ and permeability $\left(\mu_{\text {eff }}\right)$ for getting negative refractive index using metallic SRRs is investigated.

\section{PARAllel-Coupled Filter DesigN}

The first step in designing a filter is to determine the fractional bandwidth $(\delta)$ as follows [21].

$$
\delta=\frac{f_{2}-f_{1}}{f_{o}}
$$

$f_{1}, f_{2}$ and $f_{\mathrm{o}}$ are the lower-, upper- cutoff frequency and center frequency respectively.

The frequency transformation from the low-pass prototype filter to the band-pass filter is expressed as

$$
\frac{f}{f_{c}}=\frac{2}{\delta}\left(\frac{f_{i}-f_{o}}{f_{o}}\right)
$$

where $f_{\mathrm{c}}$ is the prototype cutoff frequency (equal to 1.0) and filter frequency $(f)$ has to be defined in the filter specification. A fractional bandwidth of 0.4762 and transformation ratio of -3.64 should be obtained. The second step is examining the filter prototype specification which meets the insertion loss requirements. The family of curves shown in Fig. 3 applies to Chebyshev prototypes all giving a $-0.01 \mathrm{~dB}$ pass-band ripple.

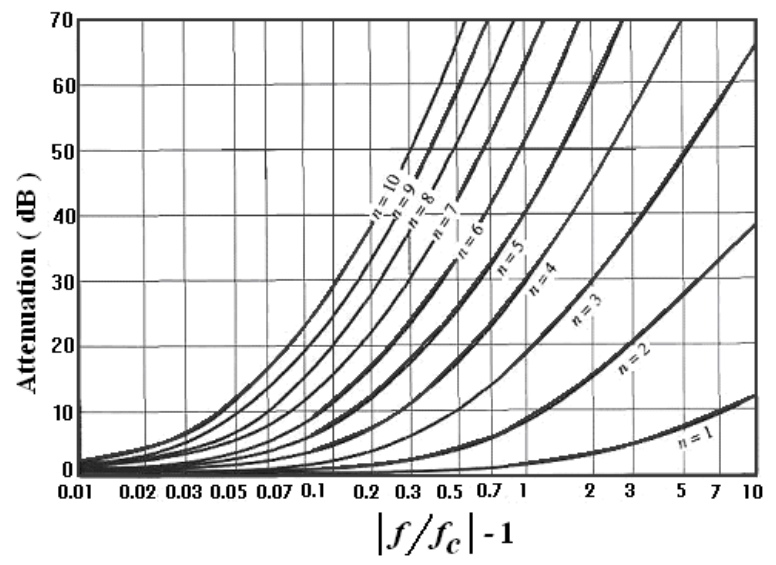

Fig. 3. Attenuation versus normalized frequency.

The independent variable has been adjusted as

$$
\left|f / f_{c}\right|-1
$$

From the Fig. 3, the value of $\left|f / f_{c}\right|-1$ is found to be 2.64 . A third order $(n=3)$ design therefore gives a $-37.5 \mathrm{~dB}$ insertion loss of $f_{i}$, this is equivalent to the $1.4 \mathrm{GHz}$ point in the final requirement. For ripple amplitude $R>0.01 \mathrm{~dB}$, only odd order designs are permissible, ensuring that $g_{\mathrm{o}}=g_{n}=1$ accurate. The equations for the calculation of element values $\left(g_{n}\right)$ are given below [19].

$$
\begin{gathered}
g_{1}=\frac{2}{\gamma} \sin \left(\frac{\pi}{2 n}\right) \\
g_{i}=\frac{1}{g_{i-1}} \frac{4 \sin \left[\frac{(2 i-1) \pi}{2 n}\right] \sin \left[\frac{(2 i-3) \pi}{2 n}\right]}{\gamma^{2}+\sin ^{2}\left[\frac{(i-1) \pi}{n}\right]}
\end{gathered}
$$

for $i=2,3, \cdots, n$

$$
g_{n+1}=\left\{\begin{array}{llll}
1.0 & \text { for } & n & \text { odd } \\
\operatorname{coth}^{2}\left(\frac{\beta}{4}\right) & \text { for } & n & \text { even }
\end{array}\right.
$$

where

$$
\begin{aligned}
& \beta=\ln \left[\operatorname{coth}\left(\frac{R}{17.37}\right)\right] \\
& \gamma=\sinh \left[\frac{\beta}{2 n}\right]
\end{aligned}
$$

Based on the design specification, a $10.5 \mathrm{GHz}$ center frequency filter is required for microwave wireless receiver 
such as satellite television receivers. The element values obtained are given in the Table II.

TABLE II: ELEMENTS VALUES OF PARALLEL-COUPLED FILTER

\begin{tabular}{|c|c|c|c|c|}
\hline $\mathrm{g}_{\mathrm{o}}$ & $\mathrm{g}_{1}$ & $\mathrm{~g}_{2}$ & $\mathrm{~g}_{3}$ & $\mathrm{~g}_{4}$ \\
\hline 1 & 1.0316 & 1.1474 & 1.0316 & 1 \\
\hline
\end{tabular}

The third step is to calculate the inverter admittances (normalized for $50 \Omega$ impedance) and hence coupled-line impedances use the following equations $[15,21]$.

$$
\begin{aligned}
& Z_{o} J_{1}=\sqrt{\frac{\pi \delta}{2 g_{o} g_{1}}} \\
& Z_{o} J_{n}=\frac{\pi \delta}{2 \sqrt{g_{n-1} g_{n}}}
\end{aligned}
$$

for $n=2,3$

$$
\begin{gathered}
Z_{o} J_{n+1}=\sqrt{\frac{\pi \delta}{2 g_{n} g_{n+1}}} \\
Z_{o e}=Z_{o}\left[1+J Z_{o}+\left(J Z_{o}\right)^{2}\right] \\
Z_{o o}=Z_{o}\left[1-J Z_{o}+\left(J Z_{o}\right)^{2}\right]
\end{gathered}
$$

where

$$
Z_{o} \approx \sqrt{Z_{o e} Z_{o o}}
$$

$J$ is the inverter, $Z_{o}$ is the system characteristic impedance, $Z_{o e}$ is the even characteristic impedance and $Z_{o o}$ is the odd characteristic impedance. All results are indicated in the Table III.

TABLE III: PARAMETERS OF PARALLEL-COUPLED FILTER
\begin{tabular}{|c|c|c|c|c|}
\hline$n$ & $Z_{o} J_{n}$ & $Z_{o e}(\Omega)$ & $Z_{o o}(\Omega)$ & $Z_{o}(\Omega)$ \\
\hline 1 & 0.8515 & 128.83 & 43.68 & 75.01 \\
\hline 2 & 0.6875 & 108 & 39.25 & 65.11 \\
\hline 3 & 0.6875 & 108 & 39.25 & 65.11 \\
\hline 4 & 0.8515 & 128.83 & 43.68 & 75.01 \\
\hline
\end{tabular}

\section{NUMERICAL AND SIMULATION RESULTS}

\section{A. Parallel-Coupled Microwave Filters}

Table I indicates the specifications of the broadband microwave parallel-coupled filter design. Using (4) - (6) to find the elements value $g_{n}$ which indicates in the table II. Then calculate the value of even and odd characteristic impedances $\left(Z_{o e}, Z_{o o}\right)$ from (10) \& (11). After finding the values of $Z_{o}$ in (12) for each element and by using standard chart [21] or tables in [22] with some approximation, we get

\begin{tabular}{|c|c|c|c|}
\hline $\begin{array}{l}\text { Element } \\
\text { Number }\end{array}$ & $\begin{array}{c}\mathrm{W} \\
(\mathrm{mm})\end{array}$ & $\begin{array}{c}\mathrm{L} \\
(\mathrm{mm})\end{array}$ & $\begin{array}{c}\mathrm{S} \\
(\mathrm{mm})\end{array}$ \\
\hline $1=8$ & 0.75 & 2 & \multirow{3}{*}{$\begin{array}{c}\mathrm{S}_{1}=\mathrm{S}_{2}=0.04 \\
\mathrm{~S}_{3}=0.076\end{array}$} \\
\hline $2=3=6=7$ & 0.169 & 3.38 & \\
\hline $4=5$ & 0.5564 & 2 & \\
\hline
\end{tabular}
the values of $w / h$ and $s / h$ for each element. The dimensions of layout design of parallel-coupled microwave band-pass filter are indicated in the Table IV.

TABLE IV: SIMULATION DIMENSIONS OF COUPLED-LINES FOR THE DESIGN IN FIGURE 4 WHICH INDICATES THE ELEMENTS NUMBER

The layout design shown in Fig. 4 has the dimensions $12.76 \mathrm{~mm} \times 3 \mathrm{~mm} \times 0.705 \mathrm{~mm}$.

The insertion and return loss at $-3 \mathrm{~dB}$ for the band $(8-13)$
$\mathrm{GHz}$ and the rejection frequencies are shown in Fig. 5. The modified design has the same dimensions of elements and spacing as compared with the first design but eliminate layout dimensions as a modified shape, which is shown in Fig. 6.

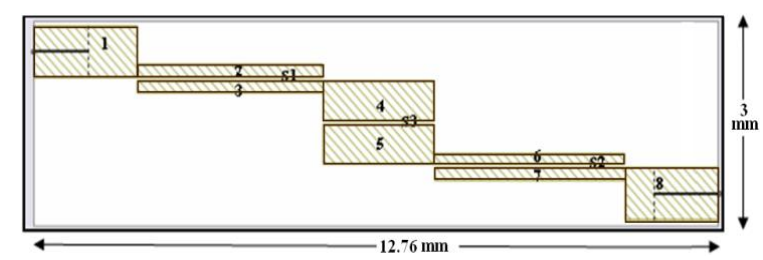

Fig. 4. Layout of broadband parallel-coupled microwave filter.

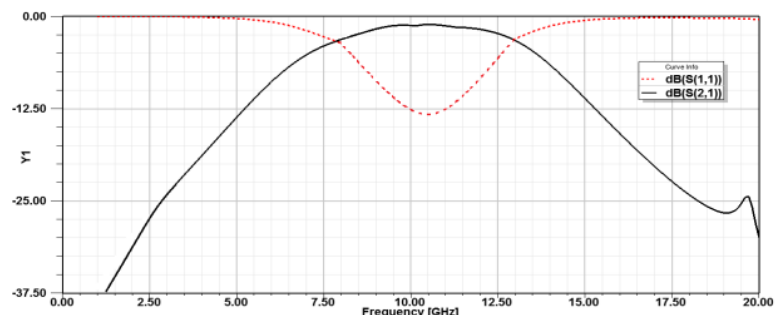

Fig. 5. Filter response simulation for broadband parallel-coupled microwave filter.

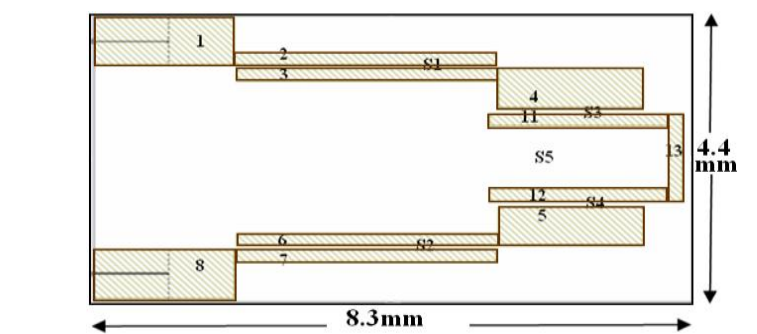

Fig. 6. Modified design of wideband parallel-coupled microwave filter.

The separation of the U-shape resonator is chosen with the aid of software (Ansoft HFSS) and listed in Table V. If the separation is chosen to be too small, there will be a lot parasitic reactance. If the separation is too big, losses will be great. Therefore a balance between the two has to be met [19].

\begin{tabular}{|c|c|c|c|}
\hline $\begin{array}{l}\text { Element } \\
\text { Number }\end{array}$ & $\begin{array}{c}\mathrm{W} \\
(\mathrm{mm})\end{array}$ & $\begin{array}{c}\mathrm{L} \\
(\mathrm{mm})\end{array}$ & $\begin{array}{c}\mathrm{S} \\
(\mathrm{mm})\end{array}$ \\
\hline $1=8$ & 0.75 & 2 & \multirow{5}{*}{$\begin{array}{c}\mathrm{S}_{1}=\mathrm{S}_{2}=0.05366 \\
\mathrm{~S}_{3}=\mathrm{S}_{4}=0.108 \\
\mathrm{~S}_{5}=0.9927\end{array}$} \\
\hline $2=3=6=7$ & 0.187 & 3.6 & \\
\hline $4=5$ & 0.59 & 2 & \\
\hline $11=12$ & 0.187 & 2.68 & \\
\hline 13 & 0.202 & 0.992 & \\
\hline
\end{tabular}
The U-shape resonator makes a shift in center frequency to become $9.5 \mathrm{GHz}$ that means miniaturize in dimensions. The overall dimensions of the modified filter are $(8.3 \mathrm{~mm} \times 4.4 \mathrm{~mm}$ $\times 0.705 \mathrm{~mm})$.

TABLE V: SimUlation DimENSIONS OF MODIFIED FILTER DESIGN

The insertion loss $\left(\mathrm{S}_{21}-\mathrm{dB}\right)$ and return loss $\left(\mathrm{S}_{11}-\mathrm{dB}\right)$ of modified filter are shown in Fig. 7. A band-pass of $7.5 \mathrm{GHz}$ bandwidth at $9.5 \mathrm{GHz}$ center frequency and stop-band $6.5 \mathrm{GHz}$ bandwidth at $16 \mathrm{GHz}$ center frequency.

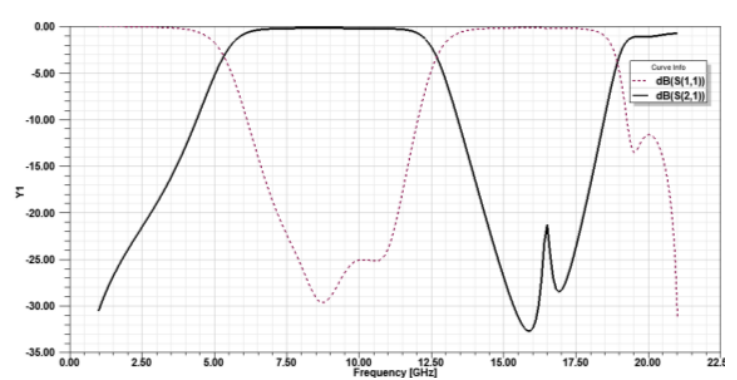

Fig. 7. Filter response simulation for modified parallel-coupled filter. 


\section{B. SRR Structure}

The metallic SRRs metamaterial are shown in Fig. 8. The split rings are copper in a square with a thickness of $0.035 \mathrm{~mm}$ and the geometric parameters $(\mathrm{a})=(\mathrm{b})$ are $1.5 \mathrm{~mm}$. In Fig. 8 -a, the parameter $(\mathrm{c})$ is $0.11 \mathrm{~mm}$, the gap between the inner and outer rings, represented by (d), is $0.12 \mathrm{~mm}$, and each of the splits in the inner and outer rings has the same width $(g)$ of $0.15 \mathrm{~mm}$. In Fig. 8-b, the parameters (c), (d) and $(g)$ are $0.15 \mathrm{~mm}, 0.05 \mathrm{~mm}$ and $0.15 \mathrm{~mm}$ respectively.

The SRR is electrically very compact due to use of sub-wavelength structure (generally $\leq \lambda / 10$ ) but in our design the sub-wavelength approximate to $\lambda / 20$.

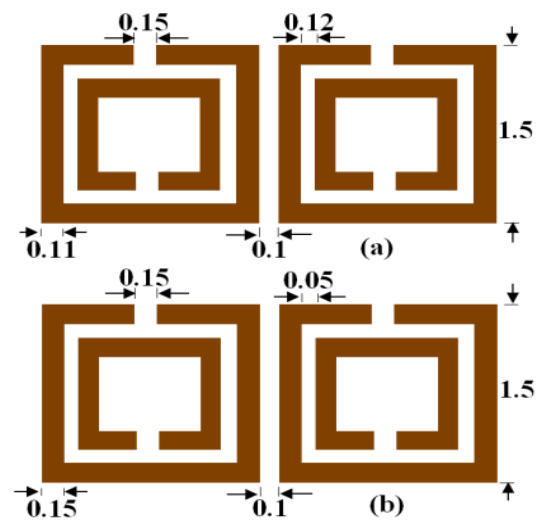

Fig. 8. Metallic SRRs metamaterial (a) at $10.5 \mathrm{GHz}$, (b) at $9.5 \mathrm{GHz}$. All values are indicated in $(\mathrm{mm})$.

The transmission coefficient $\left(\mathrm{S}_{21}-\mathrm{dB}\right)$ of the double ring metamaterial is shown in Fig. 9. It is obvious that there are a stop-band around $10.5 \mathrm{GHz}$ and $9.5 \mathrm{GHz}$ that is shown in Fig. 9 (a), and 9(b) respectively.
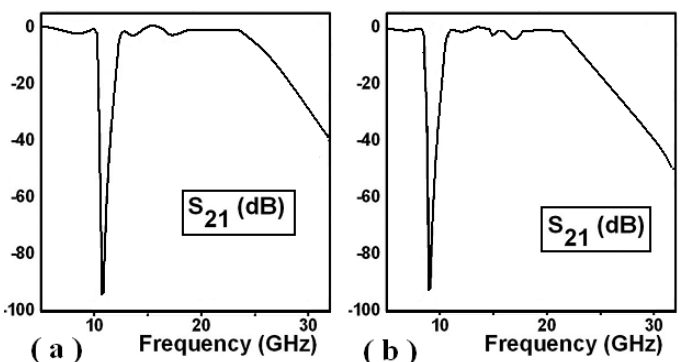

Fig. 9. Transmission coefficient of the metallic SRRs metamaterial. The stop-band at (a) $10.5 \mathrm{GHz}$, (b) $9.5 \mathrm{GHz}$.

The calculation was performed to obtain the $\varepsilon_{\text {eff }}$ and $\mu_{\text {eff }}$ from the scattering parameters $\left(\mathrm{S}_{21}\right.$ and $\left.\mathrm{S}_{11}\right)$ of the proposed SRR metamaterial [23], [24]. Fig. 10 shows the $\varepsilon_{\text {eff }}$ and $\mu_{\text {eff }}$ respect to frequency for the metallic SRRs. It is also obvious that the retrieved negative $\mu_{\text {eff }}$ and positive $\varepsilon_{\text {eff }}$ in the fundamental resonant regime are contributing to the stop-band near $10.5 \mathrm{GHz}$ and $9.5 \mathrm{GHz}$ that is shown in Fig. $10(\mathrm{a})$, and 10(b) respectively.
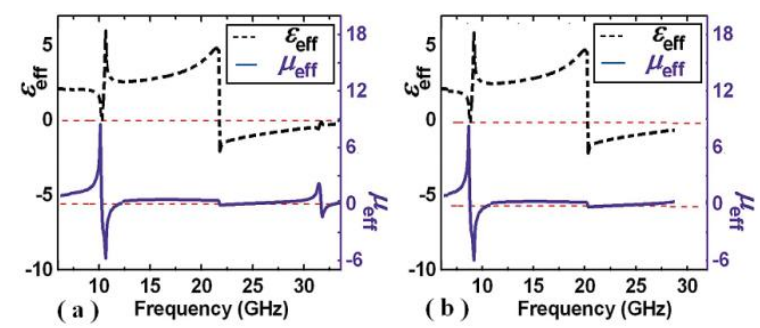

Fig. 10. Effective permittivity and permeability of the metallic SRRs metamaterial (a) stop-band near $10.5 \mathrm{GHz}$, (b) stop-band near $9.5 \mathrm{GHz}$.

\section{Metamaterial Parallel-Coupled Microwave Filters}

After adding metallic SRRs structures to our designs as shown in Fig. 11, and Fig. 13 we saw the obvious changes in responses. So, we got on stop-band at around the center frequency to cut the previous BPF to dual-band operate at $7.5 \mathrm{GHz}$ with $3 \mathrm{GHz}$ bandwidth and $12.5 \mathrm{GHz}$ with $2 \mathrm{GHz}$ bandwidth that is shown in Fig. 12.

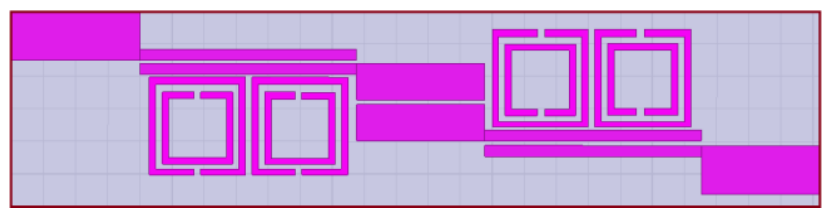

Fig. 11. Layout of metamaterial dual-band band-pass filter.

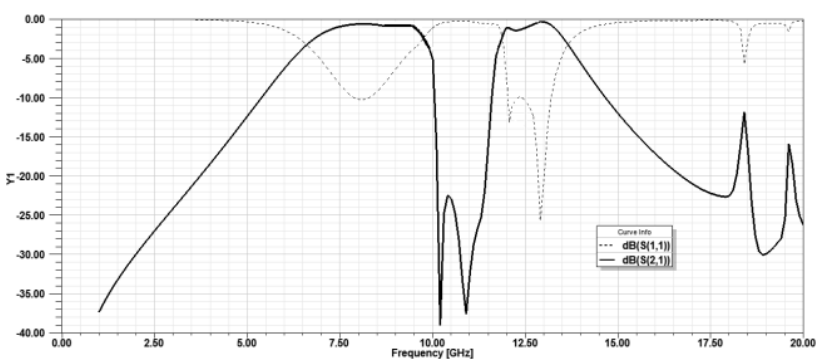

Fig. 12. The response of metamaterial dual-band band-pass filter.

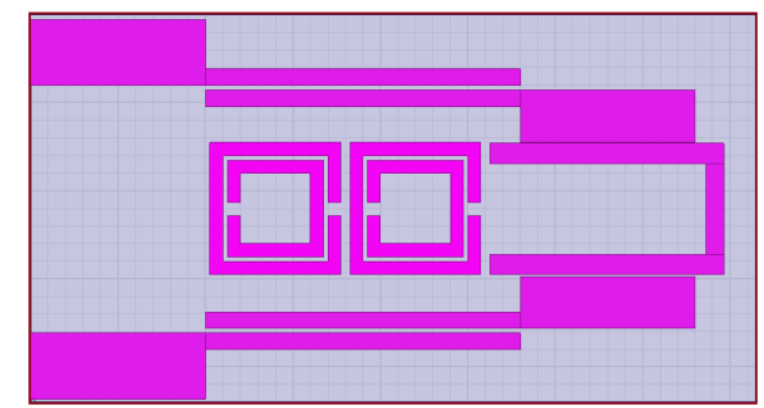

Fig. 13. Layout of modified metamaterial dual-band band-pass filter.

From Fig. 14 we got on stop-band around the certain frequency to cut the previous band-pass filter to dual-band operate at $8.2 \mathrm{GHz}$ with $2.5 \mathrm{GHz}$ bandwidth and $11 \mathrm{GHz}$ with $3.5 \mathrm{GHz}$ bandwidth.

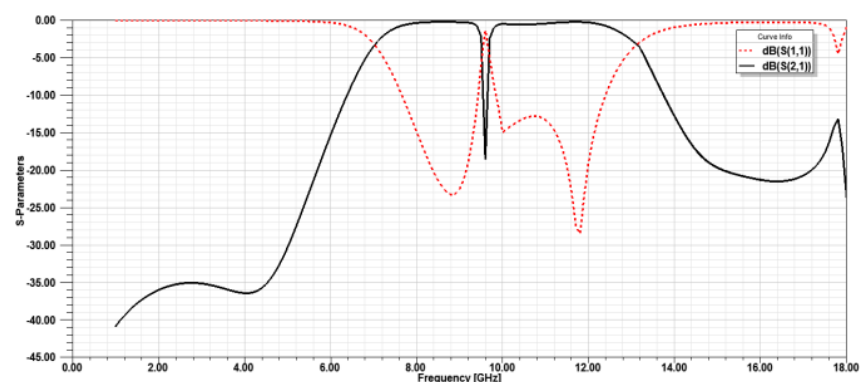

Fig. 14. The response of the modified metamaterial filter contains U- shape structure.

\section{CONCLUSIONS}

In this paper, two designs of broadband parallel- coupled microwave band-pass filters have been presented. The effect of SRRs on filters is also presented. All designs are carried out by analysis and simulation using Ansoft HFSS software. The numerical results agreed well with the simulation results. Numerical results show that a rejection level of almost $-37.5 \mathrm{~dB}$ at pass-band frequency can be obtained when using a substrate material of RT/duroid $6010, \varepsilon_{r}=10.2, \tan \alpha=0.0023$, 
$t=0.635 \mathrm{~mm}$ with input impedance equal to $50 \Omega$. Also the results in the second design (modified) are better compared to the first design. The U-shape resonator in the modified design gives wider bandwidth and also the center frequency is shifted from $10.5 \mathrm{GHz}$ to $9.5 \mathrm{GHz}$. The response of metallic SRRs metamaterial is numerically investigated. The presence of SRRs in the parallel coupled-line filters modifies the characteristics of the coupled-line sections as compared to the structures without SRRs. Therefore, it is necessary to recalculate dimensions to preserve the central filter frequency and bandwidths. Finally apply this structure (SRR) in filter design to get dual-band-pass or stop-band around the center frequency with high return loss.

\section{REFERENCES}

[1] T. S. Laverghetta, Microwave Measurements and Techniques, Second Printing, Artech House, Inc. 1978.

[2] T. S. Laverghetta, Microwaves and Wireless Simplified, 2nd Edition, Artech House, Inc. 2005, ch. 4, pp. 132-133.

[3] D. K. Misra, Radio-Frequency and Microwave Communication Circuits, John Wiely \& Sons. Inc. 2001, ch. 8 , pp. 295-296.

[4] V. G. Veselago, "The electrodynamics of substances with simultaneously negative values of $\varepsilon$ and $\mu$,"Sov. Phys. Usp, vol. 10, no. 4, pp. 509-514, 1968

[5] J. B. Pendry, A. J. Holden, and D. J. Robbins, "Magnetism from conductors and enhanced nonlinear phenomena," IEEE Trans. Microwave Theory and Tech, vol. 47, issue 11, pp. 2075-2084, 1999.

[6] T. J. Yen, W. J. Padilla, N. Fang, D. C. Vier, D. R. Smith, J. B. Pendry, D. N. Basov, and X. Zhang, "Terahertz magnetic response from artificial materials," Science, vol. 303, no. 5663, pp. 1494-1496, March 2004.

[7] H. O. Moser, B. D. F. Casse, O. Wilhelmi, and B. T. Saw, "Terahertz response of a microfabricated rod split ring resonator electromagnetic metamaterial," Phys. Rev. Lett. vol. 94, issue 6, 2005.

[8] B. D. F. Casse, H. O. Moser, J. W. Lee, M. Bahou, S. Inglish, and L. K. Jian, "Towards three-dimensional and multilayer rod-split-ring metamaterial structures by means of deep x-ray lithography," Appl. Phys. Lett. vol. 90, issue25, 2007.

[9] J. B. Pendry, A. J. Holden, and W. J. Stewart, "Extremely low frequency plasmons in metallic mesostructures," Phys. Rev. Lett, vol. 76, no. 25, pp.4773-4776, 1996.

[10] R. A. Shelby, D. R. Smith, and S. Schultz, "Experimental verification of a negative index of refraction," Science, vol. 292, Issue 5514, pp.77-79, 2001.

[11] F. Martin, J. Bonache, F. Falcone, M. Sorolla, and R. Marque's "Split ring resonator-based left-handed coplanar waveguide," Appl. Phys. Lett., vol. 83, pp. 4652 - 4654, 2003.

[12] V. M. Shalaev, "Optical negative-index metamaterials," Nature Photonics, vol. 1, pp. 41-48, 2007.

[13] C. M. Soukoulis, S. Linden, and M. Wegener, "Negative refractive index at optical wavelengths," Science, vol. 315, no. 5808, pp. 47-49, Jan. 2007.

[14] J. Garci'a-Garci'a, F. Marti'n, E. Amat, F. Falcone, J. Bonache, I. Gil, T. Lopetegi, Miguel A. G. Laso, A. Marcotegui, M. Sorolla, and R. Marque's, "Microwave filters with improved stop band based on sub-wavelength resonators," IEEE Trans. Microwave Theory Tech., vol. 53, pp. 1997 - 2006, June 2005.

[15] D. M. Pozar, Microwave Engineering, John Wiley \& Sons Ltd., 2005.

[16] C.-Y. Chang and T. Itoh, "A modified parallel-coupled filter structure that improves the upper stopband rejection and response symmetry," IEEE Trans. Microwave Theory Tech., vol. 39, no.10, pp. 310-314, Feb. 1991.

[17] M. M. Abd-Elrazak, "Design of a broadband parallel-coupled microstrip filters without spurious resonances," Active and Passive Electronic Components, vol. 2007, 2007.

[18] H. L. Gan, D. X. Yang, and D. W. Lou, "Optimized design method of microstrip parallel-coupled bandpass filters with compensation for center frequency deviation," Progress In Electromagnetics Research Symposium, vol. 1, no, 3, pp. 298-302, 2005.

[19] S. Lim, H. Pin, "Design and Modification of Parallel-Coupled Bandpass Filter," University of Newcastle, Callagham, Australia, 2002.

[20] C. J. Kikkert, "Designing low cost wideband microstrip bandpass filters," in Proc. IEEE TENCON '05 Conference, Australia, November 2005, pp. 1-6.

[21] T. C. Edwards, Foundations for Microstrip Circuit Design, John Wiley \& Sons Ltd., 1988.

[22] T. T. Ha, Solid-State Microwave Amplifier Design, Joh Widely \& Sons. Inc. 1981.

[23] D. R. Smith, D. C. Vier, Th. Koschny, and C. M. Soukoulis, "Electromagnetic parameter retrieval from inhomogeneous metamaterials," Physical Review E, vol. 71, issue 3, 2005.

[24] X. Chen, T. M. Grzegorczyk, B. I. Wu, J. Pacheco, and J. A. Kong, "Robust method to retrieve the constitutive effective parameters of metamaterials," Phys. Rev. E, vol. 70, issue 1, 2004.

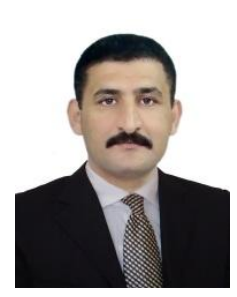

Ahmed Hameed Reja was born in Baghdad-Iraq on the eighth of January 1971. He received his BSc. Electrical and Electronics Engineering Communication Department at Al - Rasheed College of Engineering - University of Technology-Baghdad - Iraq in 1995, and M.Sc. Electrical and Electronics Engineering Communication and Microwave field from University of Technology, Baghdad - Iraq in 2002. $\mathrm{He}$ is a faculty member in the Department of Electromechanical Engineering at University of Technology. He is pursuing a Ph.D. degree since Feb. 2012 in the Department of Electronics and Communication Engineering - Jamia Millia Islamia, New Delhi - India. He has authored of several papers in International / national Journals and proceedings of conferences. His research interests in Communication and Microwave Engineering.

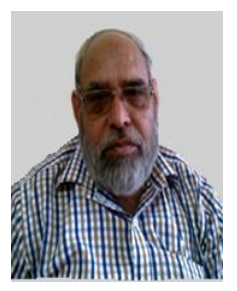

Syed Naseem Ahmad was born in New Delhi-India on the second of July 1952. He received his BSc. In Electrical and Electronics Engineering in Aligarh Muslim University, 1975, M.Sc. Electrical and Electronics Engineering at Aligarh Muslim University, 1979, and Ph.D. Engineering in Jamia Millia Islamia, New Delhi-India, 2005.

$\mathrm{He}$ is a professor in the department of Electronics and communication engineering at Jamia Millia Islamia, New Delhi- India. He has authored of several papers in different international / national Journals and proceedings of conferences. His research interests in Signal Processing, Electronics, Communication and Microwave Engineering.

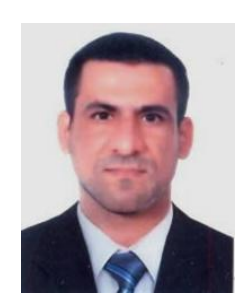

Mushtaq A. Alqaisy was born in Baghdad- Iraq on the eighth of July 1972. He received his BSc. Electrical and Electronics EngineeringCommunication Department at Al-Rasheed College of Engineering-University of Technology-Baghdad - Iraq in 1996, and M.Sc. Electrical and Electronics Engineering - Communication department from University of Technology -Baghdad-Iraq in 2005. He is pursuing a Ph.D. degree since July 2011, in the Department of Electronics and Communication Engineering Universiti Tenaga Nasional, Malaysia. He has authored of several papers in International Journals and proceedings of conferences. His research interests in communication and Microwave Engineering. 